\title{
Data Selective Rake Reception for Underwater Acoustic Communication in Strong Multipath Interference
}

\author{
Shingo Yoshizawa, ${ }^{1}$ Hiroshi Tanimoto, ${ }^{1}$ and Takashi Saito ${ }^{2}$ \\ ${ }^{1}$ Department of Electrical and Electronic Engineering, Kitami Institute of Technology, Kitami, Japan \\ ${ }^{2}$ Mitsubishi Electric TOKKI Systems Corporation, Kanagawa, Japan \\ Correspondence should be addressed to Shingo Yoshizawa; yosizawa@mail.kitami-it.ac.jp
}

Received 6 January 2017; Accepted 3 April 2017; Published 22 May 2017

Academic Editor: George S. Tombras

Copyright (c) 2017 Shingo Yoshizawa et al. This is an open access article distributed under the Creative Commons Attribution License, which permits unrestricted use, distribution, and reproduction in any medium, provided the original work is properly cited.

In underwater acoustic communication (UAC), very long delay waves are caused by reflection from water surfaces and bottoms and obstacles. Their waves interfere with desired waves and induce strong multipath interference. Use of a guard interval (GI) is effective for channel compensation in OFDM. However, a GI tends to be long in shallow-water environment because a guard time is determined by a delay time of multipath. A long GI produces a very long OFDM frame in several seconds, which is disadvantageous to a response speed of communication. This paper presents a method of keeping good communication performance even for a short GI. We discuss influence of intercarrier interference (ICI) in OFDM demodulation and propose a method of data selective rake reception (DSRake). The effectiveness of the proposed method is discussed by received signal distribution and confirmed by simulation results.

\section{Introduction}

Remotely operated underwater vehicle (ROV) and autonomous underwater vehicle (AUV) are widely used in current marine surveys $[1,2]$. Wireless communication is an important underlying technology in remote control and information gathering for ROV and AUV. Since light and electromagnetic waves have large attenuation in seawater, use of sound waves is suitable for long range communication. Underwater acoustic communication (UAC) has been studied for a long time as well as radio communication. For instance, a communication unit of single-sideband amplitude modulation (SSB-AM) was developed in the 1950s. Digital modulation schemes of spread spectrum [3, 4], OFDM [5-7], and MIMO $[8,9]$ have been studied in recent studies.

Demodulation is affected by multipath interference and Doppler in UAC, which degrade communication performance. Doppler compensation has been discussed in [1013]. We focus on the problem of multipath interference in this paper. Very long delay waves are caused by reflection from water surfaces and bottoms and obstacles. Their waves interfere with desired waves and induce strong multipath interference. For mitigation of multipath interference, OFDM with a guard interval (GI) (also named as a cyclic prefix (CP)) is adopted. As far as a delay time of multipath is less than a guard time, influence of delay waves can be expressed by channel coefficients for every frequency bin. These channel coefficients can be estimated and equalized by frequency domain equalization (FDE). Effectiveness of OFDM using a GI has been verified by sea trials in [5-7].

The drawback of using a GI is decrease of communication efficiency because a GI itself is redundant. In shallowwater environment, a long GI is required when a guard time is determined by a delay time of multipath. The delay time ranges from several milliseconds to 100 milliseconds in underwater acoustic propagation, being dependent on surrounding environments. In the sea trial presented by Berger et al. [7], the GI and FFT length were set to $48 \mathrm{~ms}$ and $491 \mathrm{~ms}$. OFDM frame duration runs up to 5.4 seconds, which would be undesirable in terms of a response speed of communication.

This paper presents a method of keeping good communication performance even for a short GI. Strong multipath interference is assumed in our study, where arrival 


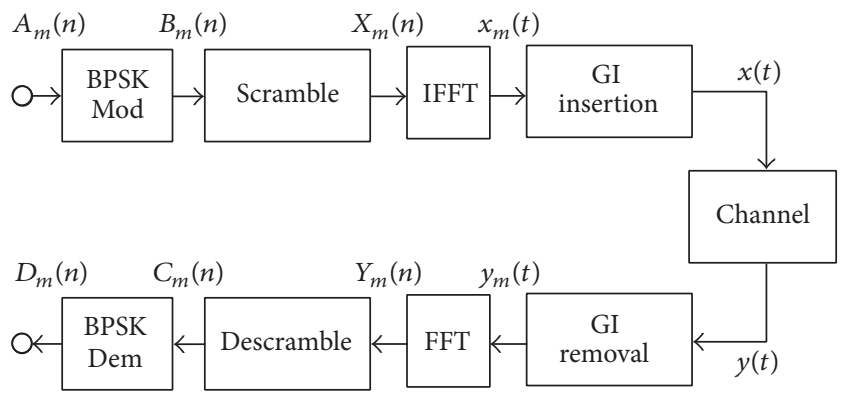

FIGURE 1: Basic OFDM model.

times of large delay waves exceed a guard time. First, we discuss the influence of interblock interference (IBI) and intercarrier interference (ICI) in received signal distribution. Although IBI always interferes with demodulation, ICI can be suppressed by taking an appropriate FFT window timing. Next, we propose a new idea of data selective rake reception (DSRake) according to the above discussion. DSRake takes multiple fingers by changing FFT window timing for every OFDM block. The best finger with the least ICI is selected by checking data errors for all fingers. With regard to QPSK modulation, the mitigation of ICI has an impact on avoiding error floor in BER performance. This paper discusses OFDM as communication scheme. As for single carrier frequency domain equalization (SC-FDE), we briefly report it in [14].

This paper is organized as follows. Section 2 discusses the influences of IBI and ICI by received signal distribution. Section 3 proposes DSRake for the mitigation of ICI. Section 4 reports simulation results evaluating DSRake in strong multipath interference. Section 5 summarizes our work.

\section{Received Signal Distribution}

2.1. OFDM Model. We discuss the influences of IBI and ICI by received signal distribution. Theoretical symbol error rates (SERs) of PSK and QAM can be obtained by probability density function (PDF) when we observe received signal amplitudes in noisy propagation channels. We use a basic OFDM model illustrated in Figure 1. In the transmitter side, all transmitted data are set to zero, given by $A_{m}(n)=0$ $(0 \leq m \leq M-1,0 \leq n \leq N-1)$. $m$ denotes a block number for $M$ OFDM blocks. $n$ is an subcarrier index for $N$ OFDM subcarriers. A transmitted symbol becomes $B_{m}(n)=1$ after BPSK modulation. The transmitted symbol is converted into 1 or -1 by multiplying random patterns of $S_{m}(n)$ in the scramble block, which becomes $X_{m}(n)$. A time-domain signal block is given by $x_{m}(t)$ after IFFT operation, where $t$ is a discrete sample time. A transmitted signal is expressed by $x(t)$ after GI insertion and parallel to serial conversion. We presuppose that this GI is given by a cyclic prefix.

In the receiver side, a received signal block of $y_{m}(t)$ is obtained by cutting out a received signal of $y(t)$ by a FFT window having a rectangular shape. A frequency domain signal block is given by $Y_{m}(n)$ after FFT operation. $C_{m}(n)$ is obtained by multiplying the random patterns of $S_{m}(n)$ used in transmitter side. Received data of $D_{m}(n)$ are obtained after

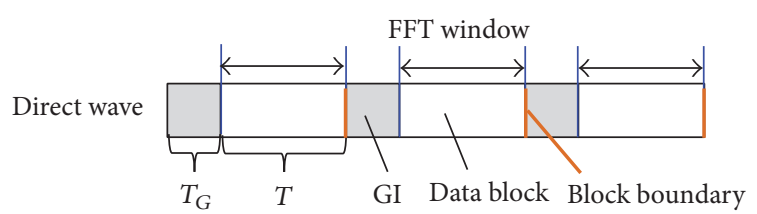

FIGURE 2: OFDM frame structure and timing positions for FFT windowing.

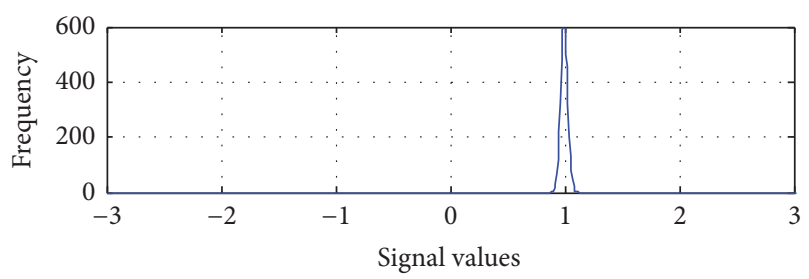

Figure 3: Received signal distribution for only direct wave.

BPSK demodulation. We set lengths of a data block, GI, and OFDM block to $T(=N), T_{G}$, and $T+T_{G}$.

We use a two-path channel model consisting of direct and delay waves. A relation between transmitted and received signals is expressed as

$$
y(t)=x(t)+\alpha x(t-\tau)+n(t)
$$

where $\alpha$ is a propagation channel coefficient $(|\alpha|<1)$ for the delay wave and $\tau$ is an arrival time difference between direct and delay waves. $n(t)$ denotes noise signal component determined by a metric of the carrier to noise ratio (CNR).

Figure 2 shows an OFDM frame structure and timing positions for FFT windowing. This figure shows the case of receiving only a direct wave. When timing synchronization is perfect, their positions are the same of those of data blocks, not overlapping with GIs. The block boundary is emphasized between OFDM blocks.

The received signal distribution for a $30-\mathrm{dB}$ CNR is shown in Figure 3. We set a data block length and a guard time to $T=256$ and $T_{G}=64$, respectively. The signal distribution for the received BPSK symbols of $C_{m}(n)$ is plotted. The total number of received BPSK symbols is $256 \times 20=5,120$. In BPSK demodulation, a symbol error occurs when $C_{m}(n)$ has a negative value. All the signals in Figure 3 locate around 1 , which indicates the error-free demodulation of $C_{m}(n) \approx$ $B_{m}(n)$.

2.2. Influence of Interblock Interference (IBI). Let us consider the influence of IBI as a long delay wave overlaps with a direct wave. Figure 4 shows the relations between direct and delay waves where their arrival time differences of $\tau=320$ and $\tau=$ 330. The propagation channel coefficient is set to $\alpha=0.7$ for a delay wave. IBI happens due to the collision of different data blocks for direct and delay waves.

The received signal distributions for Figure 4(a) are shown in Figure 5. In Figure 4(a), $(m-1)$ th block of the delay wave exactly overlaps with $m$ th block of the direct wave in the FFT window period. The received symbol of $C_{m}(n)$ can be 


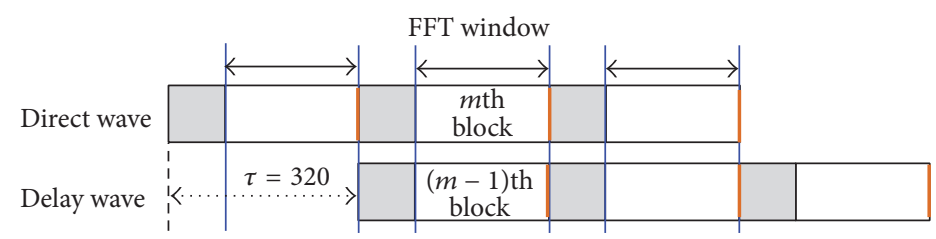

(a) $\alpha=0.7, \tau=320,30-\mathrm{dB}$ CNR

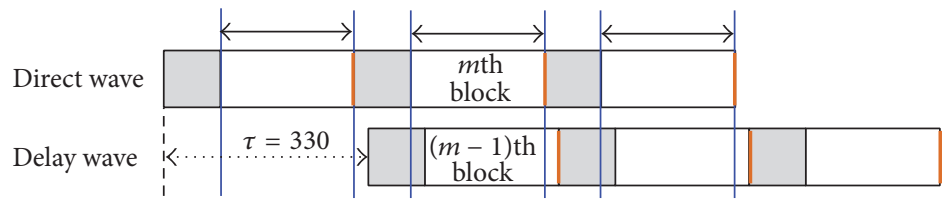

(b) $\alpha=0.7, \tau=330,30-\mathrm{dB}$ CNR

FIGURE 4: Relations between direct and delay waves $(\tau=320$ and $\tau=330)$.

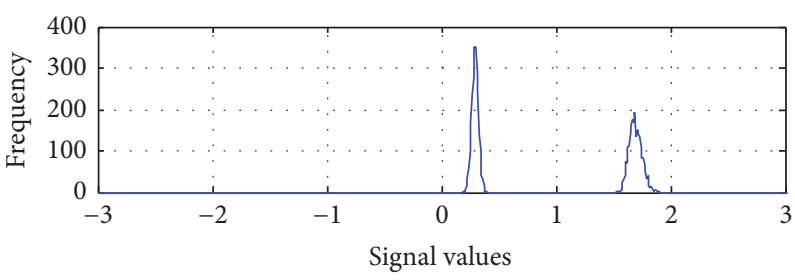

(a) $\alpha=0.7, \tau=320,30-\mathrm{dB}$ CNR

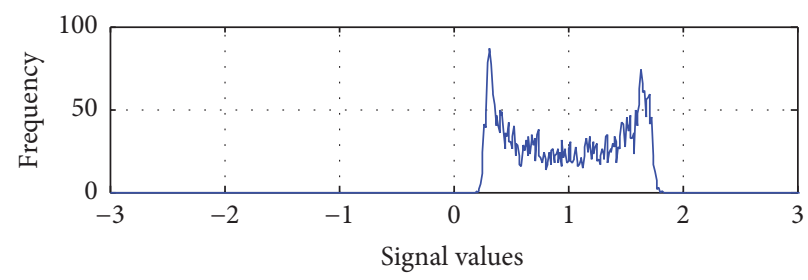

(b) $\alpha=0.7, \tau=330,30-\mathrm{dB}$ CNR

FIGURE 5: Received signal distributions for long delay waves.

introduced from the following equations, omitting the noise component of $n(t)$.

$$
\begin{aligned}
& y_{m}(t)=x_{m}(t)+\alpha x_{m-1}(t) \\
& Y_{m}(n)=X_{m}(n)+\alpha X_{m-1}(n) \\
& Y_{m}(n)=S_{m}(n) B_{m}(n)+\alpha S_{m-1}(n) B_{m-1}(n) \\
& C_{m}(n)=B_{m}(n)+\alpha S_{m}(n) S_{m-1}(n) B_{m-1}(n) . \\
& C_{m}(n)=1+\alpha S_{m}(n) S_{m-1}(n) .
\end{aligned}
$$

Since $S_{m}(n)$ and $S_{m-1}(n)$ are random patterns consisting of 1 or $-1,(6)$ gives $C_{m}(n) \in\{0.3,1.7\}$. This signal distribution can be observed in Figure 5(a). Although the signal values of $C_{m}(n)$ do not concentrate on 1 , all of them are positive. A symbol error does not occur in Figure 4(a).

In Figure 4(b), $(m-1)$ th block of the delay wave is slightly deviated from $m$ th block of the direct wave. $C_{m}(n)$ can be introduced by

$$
\begin{aligned}
& y_{m}(t)=x_{m}(t)+\alpha x_{m-1}\left(t-\tau_{d}\right) \\
& Y_{m}(n)=X_{m}(n)+\alpha X_{m-1}(n) e^{j 2 \pi n\left(\tau_{d} / N\right)} \\
& \operatorname{Re}\left[C_{m}(n)\right] \\
& \quad=\operatorname{Re}\left[B_{m}(n)+\alpha S_{m}(n) S_{m-1}(n) B_{m-1}(n) e^{j 2 \pi n\left(\tau_{d} / N\right)}\right] \\
& \operatorname{Re}\left[C_{m}(n)\right]=1+\operatorname{Re}\left[\alpha S_{m}(n) S_{m-1}(n) e^{j 2 \pi n\left(\tau_{d} / N\right)}\right],
\end{aligned}
$$

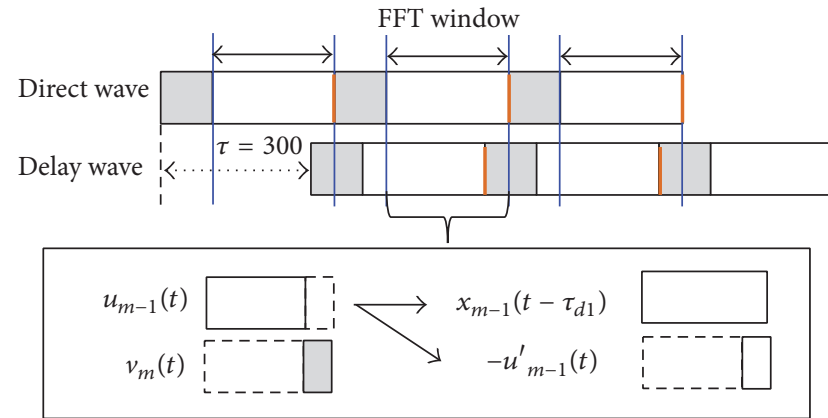

FIGURE 6: Relation between direct and delay waves $(\tau=300)$.

where we apply $\tau_{d}=\tau-T_{G}+T$ from circular shift property. The signal values of $\operatorname{Re}\left[C_{m}(n)\right]$ range from 0.3 to 1.7 as shown in Figure 5(b). This case also does not induce a symbol error.

The IBI does not take a symbol error as long as a high CNR condition is kept as for this observation. The same phenomenon would be observed even in QPSK transmission. Improvement of SNR using antenna arrays is practical rather than keeping a high CNR, where Zheng presented MRC diversity in SIMO-OFDM as a measure against insufficient guard interval in [15].

2.3. Influence of Intercarrier Interference (ICI). Let us consider the influence of ICI by giving another arrival time difference of $\tau=300$. The relation between direct and delay waves is shown in Figure 6. Different from Figure 4, $(m-1)$ th 


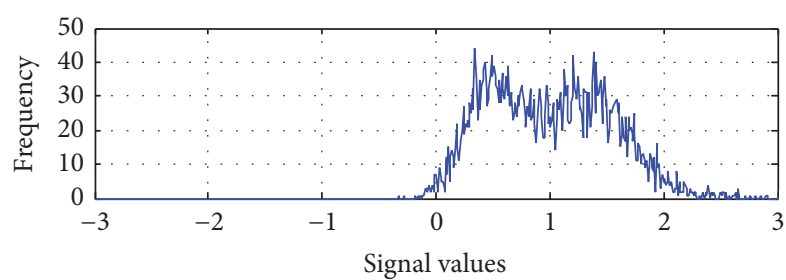

FIGURE 7: Received signal distribution affected by ICI.

data block and $m$ th GI of the delay wave overlap with $m$ th data block of the direct wave. This signal distribution is shown in Figure 7. Since some of $C_{m}(n)$ have a negative value, a symbol error occurs.

We introduce $C_{m}(n)$ as well as Section 2.2. First, the received signal of $y_{m}(t)$ is given by

$$
y_{m}(t)=x_{m}(t)+\alpha\left(u_{m-1}(t)+v_{m}(t)\right) .
$$

We decompose a received signal of the delay wave into $u_{m-1}(t)$ and $v_{m}(t)$ as shown in Figure 6 . Their functions are given by

$$
\begin{gathered}
u_{m-1}(t)= \begin{cases}x_{m-1}\left(t-\tau_{d 1}\right) & \text { if } 0 \leq t \leq \tau_{d 1}-1 \\
0 & \text { if } \tau_{d 1} \leq t \leq N-1\end{cases} \\
v_{m}(t)= \begin{cases}0 & \text { if } 0 \leq t \leq \tau_{d 1}-1 \\
x_{m}\left(t-\tau_{d 2}\right) & \text { if } \tau_{d 1} \leq t \leq N-1,\end{cases}
\end{gathered}
$$

where we apply $\tau_{d 1}=\tau-T_{G}$ and $\tau_{d 2}=\tau_{d 1}-T_{G}$ from circular shift property. $u_{m-1}(t)$ can be replaced with $x_{m-1}\left(t-\tau_{d 1}\right)-$ $u_{m-1}^{\prime}(t) \cdot u_{m-1}^{\prime}(t)$ is given by

$$
u_{m-1}^{\prime}(t)= \begin{cases}0 & \text { if } 0 \leq t \leq \tau_{d 1}-1 \\ x_{m-1}\left(t-\tau_{d 1}\right) & \text { if } \tau_{d 1} \leq t \leq N-1\end{cases}
$$

$C_{m}(n)$ can be expressed as

$$
\begin{aligned}
y_{m}(t)= & x_{m}(t)+\alpha x_{m-1}\left(t-\tau_{d 1}\right) \\
& +\alpha\left(-u_{m-1}(t)+v_{m}(t)\right) \\
\operatorname{Re}\left[C_{m}(n)\right]= & +\operatorname{Re}\left[\alpha S_{m}(n) S_{m-1}(n) e^{j 2 \pi n\left(\tau_{d 1} / N\right)}\right] \\
& +\operatorname{Re}\left[\alpha S_{m}(n)\left(-U_{m-1}^{\prime}(n)+V_{m}(n)\right)\right] .
\end{aligned}
$$

The received signal distribution of (15) would be almost the same as that of (10) if $U_{m-1}^{\prime}(n)$ and $V_{m}(n)$ are excluded.

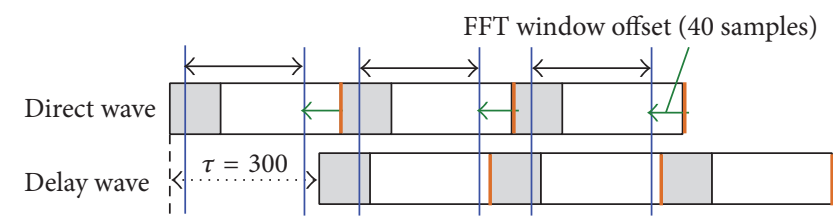

FIGURE 8: Adjustment of FFT windowing.

$U_{m-1}^{\prime}(n)$ and $V_{m}(n)$ can be expressed by using inverse discrete Fourier transform (IDFT) and DFT as

$$
\begin{aligned}
& U_{m-1}^{\prime}(n) \\
& =\sum_{t=\tau_{d 1}}^{N-1}\left[\frac{1}{N} \sum_{n=0}^{N-1} S_{m-1}(n) e^{j(2 \pi t n / N)} e^{-j\left(2 \pi \tau_{d 2} n / N\right)}\right] \\
& \cdot e^{-j(2 \pi n t / N)} \\
& V_{m}(n)=\sum_{t=\tau_{d 1}}^{N-1}\left[\frac{1}{N} \sum_{n=0}^{N-1} S_{m}(n) e^{j(2 \pi t n / N)} e^{-j\left(2 \pi \tau_{d 2} n / N\right)}\right] \\
& \cdot e^{-j(2 \pi n t / N)} .
\end{aligned}
$$

The interferences of (16) and (17) are added for every subcarrier, which corresponds to ICI. Assuming that the average amplitude for the OFDM transmit signals after IDFT is $1 / N$ (i.e., calculation within the square bracket in (16)), the average of deviations caused by $U_{m-1}^{\prime}(n)$ and $V_{m}(n)$ is roughly calculated as

$$
\pm 2 \alpha \frac{N-\tau_{d 1}}{N} \simeq \pm 0.11
$$

These deviations would be observed by comparing the received signal distributions in Figures 5 and 7 . The difference between Figures 4 and 6 is whether a block boundary is included within a FFT window.

2.4. Adjustment of FFT Window. The ICI can be avoided by changing FFT window timings, whose adjustment is illustrated in Figure 8. The time positions of FFT windows have been shifted by 40 samples ahead. The block boundaries for the delay wave are not included for their FFT windows. Although this adjustment induces a phase rotation after FFT operation in frequency domain, the phase rotation can be detected and compensated by FDE. The received signal distribution after the FFT window adjustment is shown in Figure 9, where the phase rotation can be compensated before descramble. This distribution looks like Figure 5(b) owing to the ICI avoidance.

\section{Data Selective Rake Reception (DSRake)}

The ICI avoidance is achieved when the arrival time of delay wave is perfectly known. Note that arrival times of individual delay waves are almost unknown in the actual environment. We introduce an OFDM rake reception as an alternative method, whose scheme is shown in Figure 10. Since the arrival 


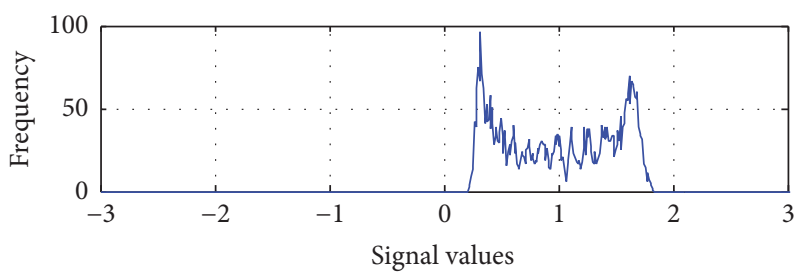

FIGURE 9: Received signal distribution after FFT window adjustment.

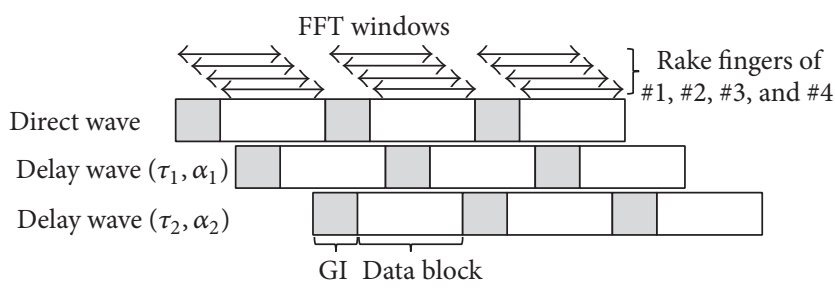

FIGURE 10: OFDM rake reception.

times $\left(\tau_{1}\right.$ and $\left.\tau_{2}\right)$ and magnitude $\left(\alpha_{1}\right.$ and $\left.\alpha_{2}\right)$ of delay waves are unknown, we take multiple FFT window timings for OFDM demodulation, that is, rake fingers.

Original rake reception itself is used as path diversity in spread spectrum [16]. In general, OFDM and rake reception for path diversity are not compatible. Received symbols in rake fingers have high correlation with each other as far as multipath delay time is less than a guard time. The improvement of received SNR is little considering increase of computational complexity in demodulation. We use the rake reception to find the best rake finger that is not affected by ICI so much. It does not aim at path diversity. The selection of rake fingers is achieved by checking data errors after demodulation, where the proposed scheme of data selective rake reception (DSRake) is shown in Figure 11. In the transmitter side, cyclic redundancy check (CRC) codes are inserted in binary data before forward error correcting (FEC) coding. In the receiver side, multiple OFDM demodulators accept received signals in rake fingers and output decoded data blocks. The best data block having no error is selected as final data by observing the CRC results in the data selection unit. If all fingers have data errors, the final data are generated by merging all decoded data in bit level.

DSRake would not be adopted in general OFDM systems such as IEEE802 WLANs and LTE in RF communication due to considerable increase in computational complexity. Note that the bandwidth of UAC is much narrower than that of RF. The increase of computational complexity for UAC does not become a problem from the viewpoint of implementation in $\mathrm{RF}$. The overhead of CRC is trivial because its length is enough for 16 bits (CRC-16) in typical usage.

DSRake belongs to selection combining (SC) in diversity combining. Maximal ratio combining (MRC) should be discussed as another method. The alternative scheme of MRC rake reception (MRCRake) is shown in Figure 12. The received symbols in rake fingers are synthesized after OFDM demodulation. Generally, a diversity gain of MRC is higher than that of SC. However, MRCRake is inferior to DSRake in
TABLE 1: Results of delay profiles.

(a) 8-m distance

\begin{tabular}{lcccc}
\hline & ch1 & ch2 & ch3 & ch4 \\
\hline Ave. delay time [ms] & 4.1 & 3.3 & 3.6 & 2.8 \\
RMS delay spread [ms] & 9.4 & 8.1 & 8.7 & 7.4 \\
\hline
\end{tabular}

(b) 20-m distance

\begin{tabular}{lcccc}
\hline & ch1 & ch2 & ch3 & ch4 \\
\hline Ave. delay time [ms] & 6.6 & 5.8 & 5.2 & 5.9 \\
RMS delay spread [ms] & 12.1 & 11.0 & 10.5 & 11.4 \\
\hline
\end{tabular}

terms of the mitigation of ICI. The synthesis of rake fingers takes in undesirable received symbols affected by ICI and the effect is limited. The superiority of DSRake will be confirmed by our simulation in the next section.

\section{Simulation}

4.1. Channel Model. As an example of underwater acoustic propagation, we use two channel models measured in a swimming pool. The delay profiles were measured on the condition of horizontal link where one transmitter and four receiver hydrophones horizontally face each other. The location of hydrophones is drawn in Figure 13. The pool length and width are $25 \mathrm{~m}$ and $13 \mathrm{~m}$ and the water depth is $1.2 \mathrm{~m}$. The distances between transmitter and receiver hydrophones are $8 \mathrm{~m}$ and $20 \mathrm{~m}$. The space of four hydrophones is $5 \mathrm{~cm}$.

The delay profiles for $8 \mathrm{~m}$ and $20 \mathrm{~m}$ distances are shown in Figures 14 and 15. A direct wave is located at 0 on the time axis and has normalized magnitude of $0 \mathrm{~dB}$. Delay waves are expressed by individual values of relative magnitude and delay time. Several clusters of delay waves are periodically observed around 30 to $35 \mathrm{~ms}, 65$ to $70 \mathrm{~ms}$, and 97 to $102 \mathrm{~ms}$ in Figure 15. These clusters come from several round trip reflections at the side walls. The delay waves of more than $-10 \mathrm{~dB}$ (i.e., less than $10 \mathrm{~dB}$ in desired to undesired signal ratio (DUR)) range from 0 seconds to $35 \mathrm{~ms}$. Since we set a guard time to $12.8 \mathrm{~ms}$ in our simulation, the delay waves beyond the GI induce IBI and ICI. If a guard time is more than $110 \mathrm{~ms}$ (i.e., more than $20 \mathrm{~dB}$ DUR), the influences of IBI and ICI would be small. However, we must keep in mind that a long GI is undesirable in terms of a response speed of communication.

Summary of the delay profiles is reported in Table 1. The $20 \mathrm{~m}$ distance shows larger values in average delay time and RMS delay spread than the $8 \mathrm{~m}$ distance. The results of average delay time and RMS delay spread are different among receiver channels to some extent. The signal correlation among received antennas would not be very high as having different propagations. Space diversity using antenna arrays is effective to improve a received SNR in this case. RMS delay spread is helpful in the determination of a GI length as long as the magnitude of delay waves is exponentially decaying. However, the magnitude of delay waves does not always fade as time goes on as shown in Figures 14 and 15. Even though the RMS delay spread is less than the GI length, the strong interference of delay waves should be considered. 


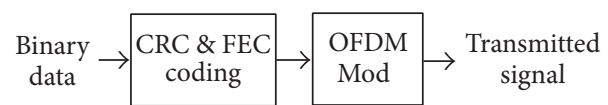

(a) Transmitter

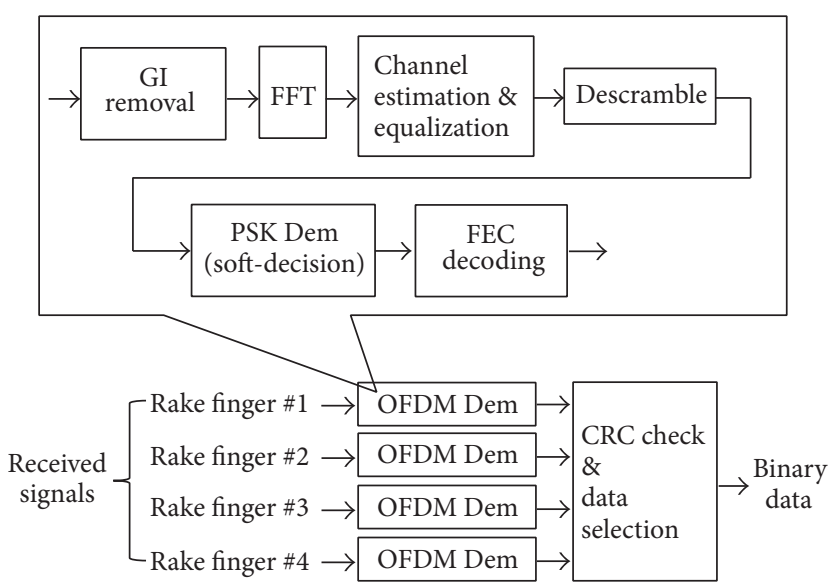

(b) Receiver

FIGURE 11: Data selective rake reception (DSRake).

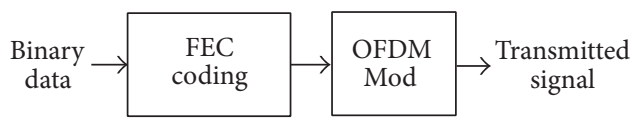

(a) Transmitter

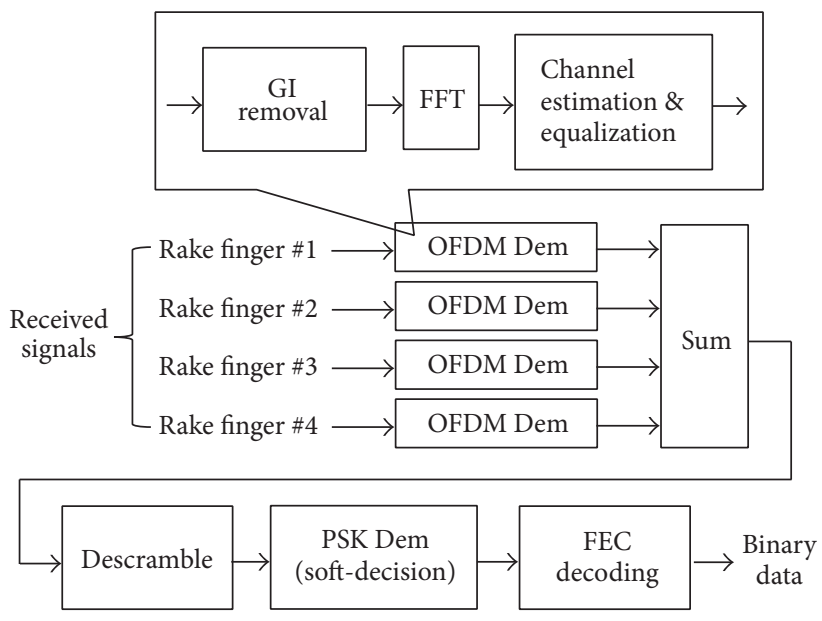

(b) Receiver

FIGURE 12: MRC rake reception (MRCRake).

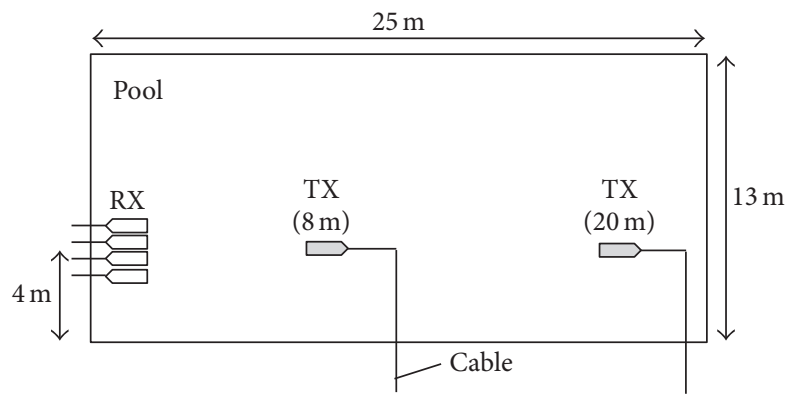

FIGURE 13: Location of transmitter and receiver hydrophones.

4.2. Simulation Parameters. The simulation parameters are enumerated in Table 2. The baseband OFDM signals with a frequency band of $-10 \mathrm{kHz}$ to $10 \mathrm{kHz}$ are modulated by a carrier wave of $50 \mathrm{kHz}$. One-tap frequency domain linear equalization based on MMSE criterion is used in channel equalization. The GI length is set to $12.8 \mathrm{~ms}$, corresponding to 256 samples in baseband domain. Two training data blocks are added to the beginning of an OFDM frame, where the frame format is shown in Figure 16. The two long training fields (LTFs) are used for channel estimation. Since the LTFs are located at the head of frame, they do not have the influence of IBI and ICI. The number of rake fingers is set to 64 for DSRake and MRCRake. We have used convolutional coding with a coding rate of $1 / 2$. The transmit data rate is about $13.3 \mathrm{kbps}$ considering the overhead of LTFs and GIs. Although the overhead of CRC codes (CRC-16) might be counted for DSRake, this overhead is very small (less than $2 \%$ ).

We apply space diversity using array antennas for the mitigation of IBI. The scheme of OFDM space diversity is shown in Figure 17. Space diversity combining based on MRC is performed after channel equalization. Space diversity combining and OFDM rake reception of DSRake or MRCRake are compatible. The diversity block is inserted into the OFDM demodulation units in Figures 11 and 12. 

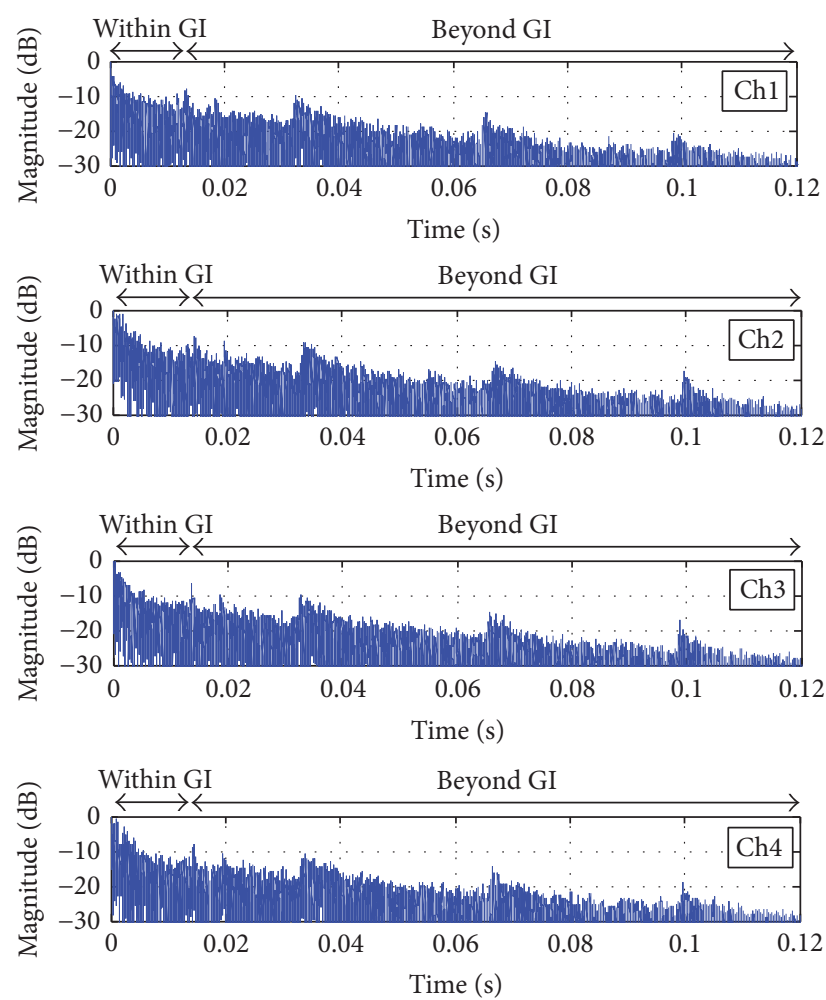

FIGURE 14: Delay profile for $8 \mathrm{~m}$ distance.
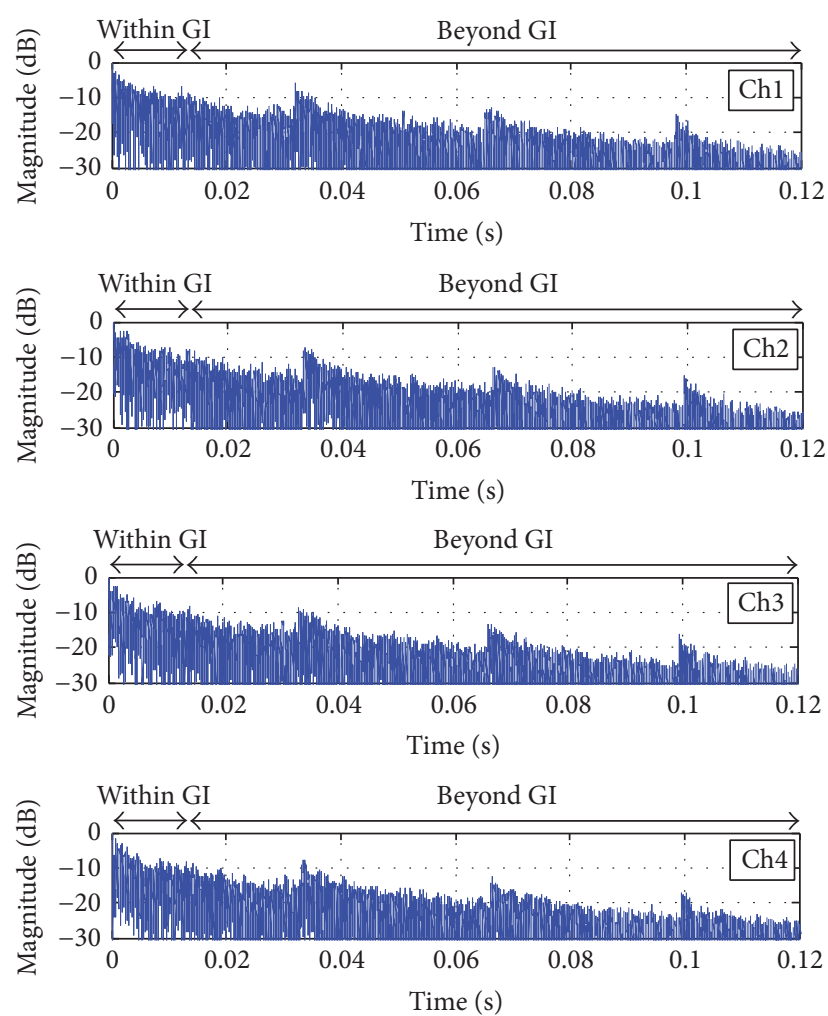

FIGURE 15: Delay profile for $20 \mathrm{~m}$ distance.

TABLE 2: Simulation parameters.

Modulation

Sampling frequency $[\mathrm{kHz}]$

Center frequency $[\mathrm{kHz}]$

Frequency band $[\mathrm{kHz}]$

FFT size

Number of data subcarriers

OFDM symbol length [ms]

GI [ms]

Number of OFDM symbols

Number of training OFDM symbols

OFDM frame length [ms]

OFDM frame data size [bytes]

FEC

Coding rate

Number of antennas

Number of OFDM rake fingers

Timing synchronization

Number of evaluated OFDM frames

\section{QPSK-OFDM}

200

50

40 to 60

1024

1024

51.2

12.8

10

2

768

1280

Convolutional coding \& Viterbi decoding

0.5

$1(\mathrm{TX}) / 4(\mathrm{RX})$

64

Perfect

100

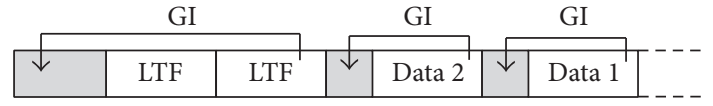

FIGURE 16: OFDM frame format.

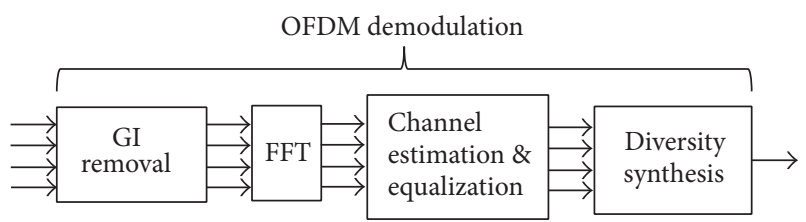

FIGURE 17: OFDM space diversity.

4.3. Simulation Results. Bit error rates (BERs) for the $8 \mathrm{~m}$ and $20 \mathrm{~m}$ distances are plotted in Figures 18 and 19. We have evaluated the schemes of single channel reception (average of four channels), space diversity, DSRake, and MRCRake. Both DSRake and MRCRake are given by the combination of space diversity and rake reception. The single channel reception has the BER floor of $10^{-2}$ due to strong multipath interference. The space diversity decreases the BER floor from $10^{-2}$ to $10^{-3}$ as shown in both figures. The influence of IBI would be decreased by space diversity combining to some extent. DSRake and MRCRake show further improvement of decreasing BER floor. DSRake is clearly superior to MRCRake from the BER results. The ICI mitigation contributes to the improvement of communication quality rather than taking path diversity. DSRake can eliminate a BER floor for the $8 \mathrm{~m}$ distance and decrease by up to $2 \times 10^{-4}$ for the $20 \mathrm{~m}$ distance. 


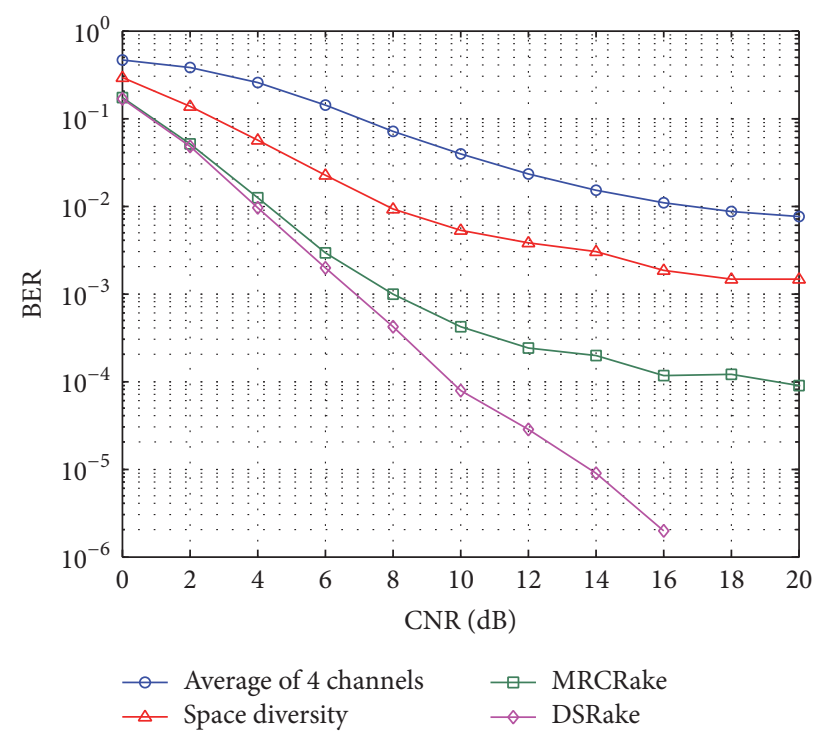

FIGURE 18: BER results for $8 \mathrm{~m}$ distance.

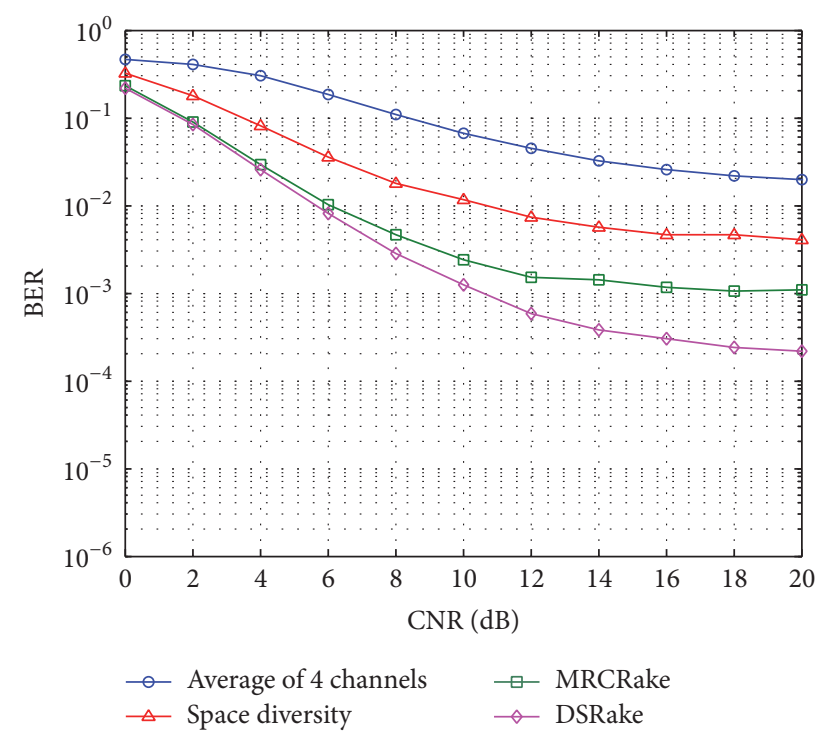

Figure 19: BER results for $20 \mathrm{~m}$ distance.

The effectiveness of DSRake in strong multipath interference has been observed from this simulation.

\section{Conclusion}

This paper presents a new method of OFDM rake reception in strong multipath interference. Very long delay waves beyond GI induce IBI and ICI. The influence of IBI and ICI is discussed by received signal distribution. Regarding ICI, we reported that the ICI avoidance can be achieved by changing FFT window timing. According to the idea of ICI avoidance, we have proposed DSRake as one of rake reception techniques. Original rake reception is used for obtaining path diversity. However, our rake reception aims at the mitigation of ICI. We have explained that selection combining by DSRake is superior to maximal ratio combining by MRCRake. The effectiveness of DSRkae has been confirmed by the simulation results based on actual underwater propagation models. In our future work, we will investigate communication performance of DSRake when Doppler effect is added.

\section{Conflicts of Interest}

The authors declare that there are no conflicts of interest regarding the publication of this paper.

\section{Acknowledgments}

The authors would like to thank the staff of Kitami City Board of Education. This work was supported by JSPS KAKENHI Grants nos. 16K18099 and 15K06048.

\section{References}

[1] L. E. Freitag and J. A. Catipovic, "A signal processing system for underwater acoustic ROV communication," in Proceedings of the 6th International Symposium on Unmanned Untethered Submersible Technology Technology, pp. 34-41, June 1989.

[2] J. Borden and J. Dearruda, "Long range acoustic underwater communication with a compact AUV," in IEEE OCEANS, October 2012.

[3] G. Loubet, V. Capellano, and R. Filipiak, "Underwater spreadspectrum communications," in Proceedings of the MTS/IEEE Conference OCEANS, pp. 574-579, October 1997.

[4] K. G. Kebkal and R. Bannasch, "Implementation of a sweepspread function for communication over underwater acoustic channels," in Proceedings of the MTS/IEEE Conference and Exhibition OCEANS, vol. 3, pp. 1829-1837, October 2000.

[5] C. M. Anil, Underwater acoustic communications in warm shallow water channels, Thesis, Doctor of Philosophy, National University of Singapore, 2006.

[6] F. Frassati, C. Lafon, P. Laurent, and J. Passerieux, "Experimental assessment of OFDM and DSSS modulations for use in littoral waters underwater acoustic communications," in Proceeding of the Europe Oceans, vol. 2, pp. 826-831, June 2005.

[7] C. R. Berger, J. Gomes, and J. M. F. Moura, "Sea-trial results for cyclic-prefix OFDM with long symbol duration," in IEEE OCEANS, June 2011.

[8] S. Roy, T. M. Duman, V. McDonald, and J. G. Proakis, "High-rate communication for underwater acoustic channels using multiple transmitters and space-time coding: receiver structures and experimental results," IEEE Journal of Oceanic Engineering, vol. 32, no. 3, pp. 663-688, 2007.

[9] P. Bouvet and A. Loussert, "An analysis of MIMO-OFDM for shallow water acoustic communications," in Proceedings of the IEEE OCEANS, pp. 1-5, September 2011.

[10] B. S. Sharif, J. Neasham, O. R. Hinton, and A. E. Adams, "A computationally efficient doppler compensation system for underwater acoustic communications," IEEE Journal of Oceanic Engineering, vol. 25, no. 1, pp. 52-61, 2000.

[11] B. Li, S. Zhou, M. Stojanovic, L. L. Freitag, and P. Willett, "Multicarrier communication over underwater acoustic channels with nonuniform Doppler shifts," IEEE Journal of Oceanic Engineering, vol. 33, no. 2, pp. 198-209, 2008. 
[12] C.-H. Hwang, K.-M. Kim, S.-Y. Chun, and S.-K. Lee, "Doppler estimation based on frequency average and remodulation for underwater acoustic communication," International Journal of Distributed Sensor Networks, vol. 2015, Article ID 746919, 8 pages, 2015.

[13] A. E. Abdelkareem, B. S. Sharif, C. C. Tsimenidis, and J. A. Neasham, "Compensation of linear multiscale doppler for OFDM-based underwater acoustic communication systems," Journal of Electrical and Computer Engineering, vol. 2012, 16 pages, 2012.

[14] S. Yoshizawa, H. Tanimoto, and T. Saito, "SC-FDE vs OFDM: performance comparison in shallow-sea underwater acoustic communication," in Proceedings of the IEEE International Symposium on Intelligent Signal Processing and Communication Systems (ISPACS '16), 5 pages, October 2016.

[15] L. T. Phuc, Y. Zheng, and Y. Karasawa, "A simplified propagation channel model for evaluating MRC diversity characteristics in SIMO OFDM with insufficient guard interval," in Proceedings of the United States National Committee of URSI National Radio Science Meeting (USNC-URSI NRSM '16), usa, January 2016.

[16] J.-H. Son, E.-H. Jeon, K.-M. Kim, D.-W. Lee, and T.-D. Park, "Alternative approach for combination of fingers in underwater acoustic communication," International Journal of Distributed Sensor Networks, vol. 2016, 16 pages, 2016. 


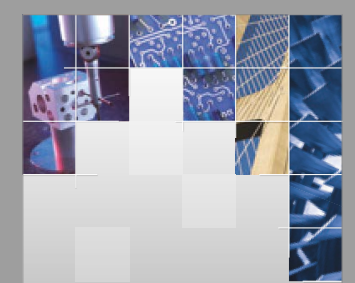

\section{Enfincering}
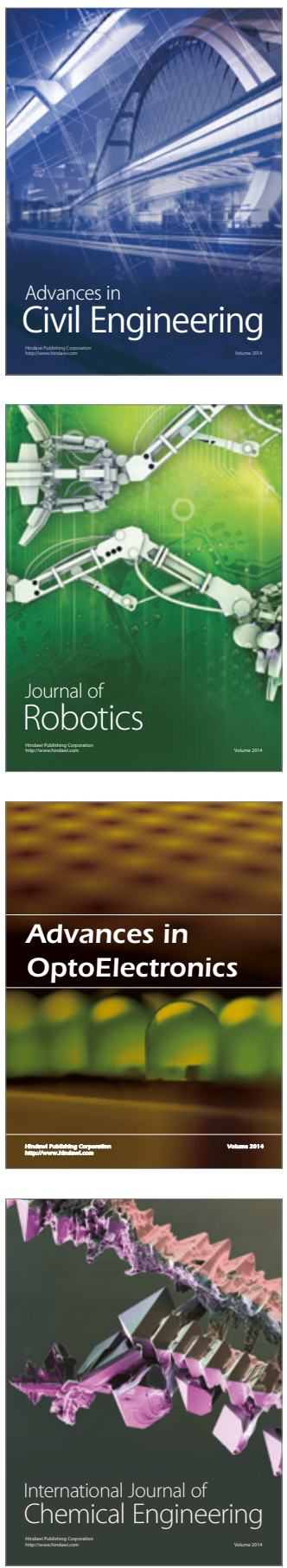

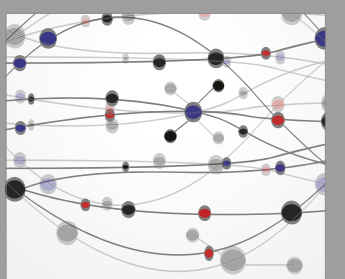

The Scientific World Journal

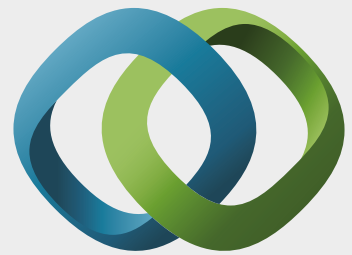

\section{Hindawi}

Submit your manuscripts at

https://www.hindawi.com
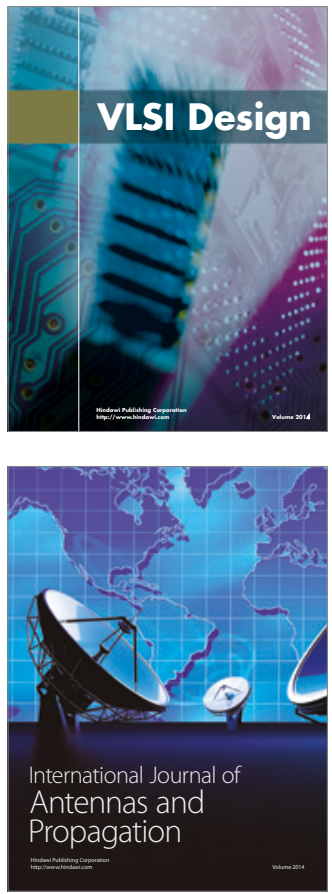

\section{Rotating}

Machinery
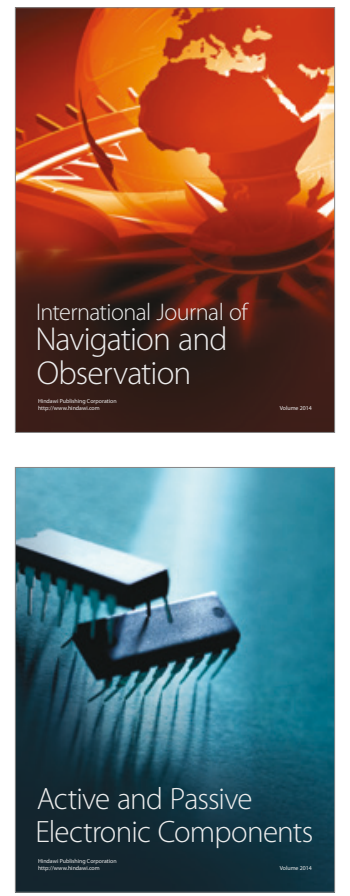
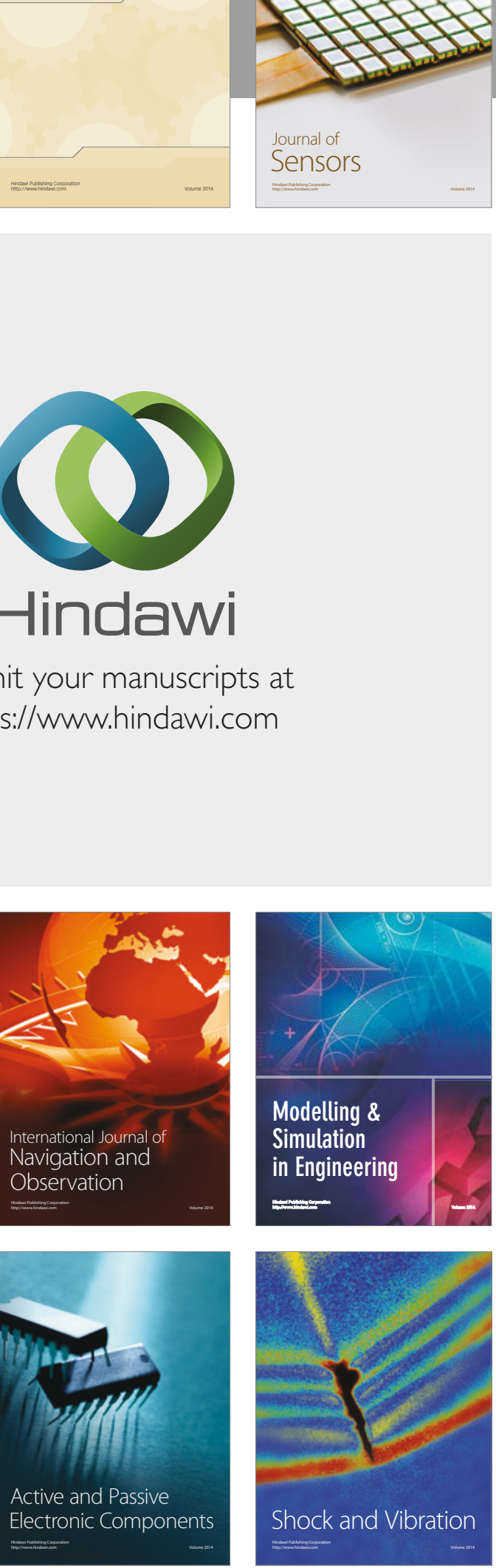
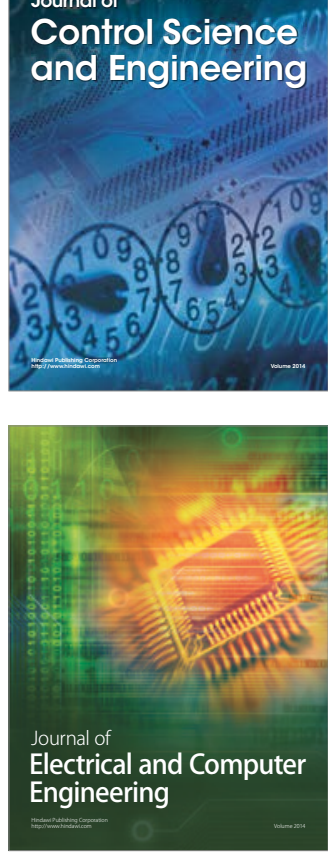

Distributed

Journal of

Control Science

and Engineering
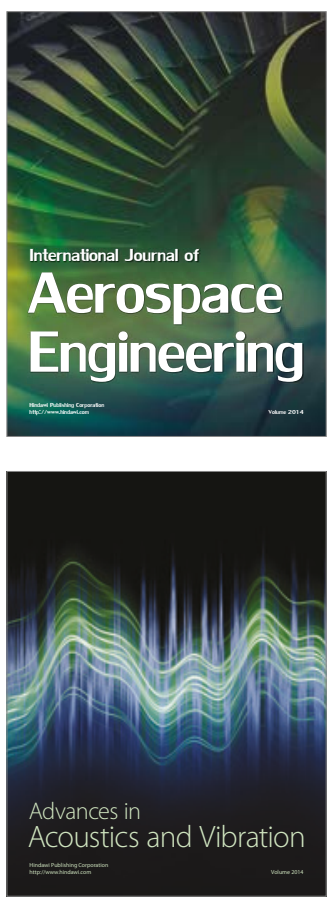

Sensor Networks 